question. The author's reasons for following this method are not apparent, but we are tempted to believe that it is in order to conform with the regulations of the University of London, which still insist that the pass degree in physics should be obtainable without a knowledge of the calculus.

The earlier chapters of the book are devoted to the principles of mechanics, special attention being paid to oscillatory and gyrostatic motion. The simpler parts of the subject seem somewhat curtailed, but that is, perhaps, excusable in view of the very comprehensive nature of the book. Following chapters on gravitation and elasticity, we find surface tension very fully treated, and it is noticeable that the author very properly points out the inaccuracy of the statement often made, that the surface tension is equal to the surface energy per unit area. Chapters xi. to xiv. introduce subjects the inclusion of which renders this book unique. The author deals in a simple yet detailed and exact manner with the motion of fluids, including applications to the properties of vortex filaments and the waves on the surfaces of liquids. In the concluding chapter there is also to be found much that is new from the student's point of view. The consideration of the kinetic theory of gases is more complete in many respects than that which has usually been given, particularly with respect to the influence of the finite size of the molecules and the various methods of estimating their size and number.

As in Mr. Edser's other books in this series, the printing is excellent, and the heavy type used in the more important statements is very desirable. Good figures and diagrams form another pleasing feature. A wealth of examples (to which answers are given) should enable the student to become thoroughly acquainted with the principles described, and there is little doubt that the book, both from its general excellence and unique features, and notwithstanding the calculus methods referred to, will become almost indispensable to the students of physics.

\section{MARINE ENGINES.}

Marine Engine Design, including the Design of Turning and Reversing Engines. By Prof. Edward M.

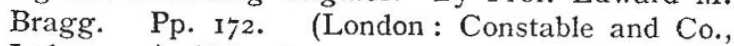
Ltd., I9II.) Price $8 s$. net.

I $\mathrm{N}$ this small book the author has endeavoured to describe methods by which the

"detailed design of the principal parts of marine engines can be determined, and has concentrated the results of several years of experience in teaching the subject of marine engine design to students of the University of Michigan."

Within these modest limits the volume should prove useful as a class-book for students, although it cannot be admitted that Mr. Bragg is accurate in the opinion, expressed in his preface, that

" most of the text-books on the subject of marine engines deal only in a general way with the subject of design, and particularly ... as referring to the numerous coordinating parts of the modern marine engine."

On the contrary, many excellent text-books existin English, French, and German-which contain full NO. 2 I92, VOL. 88] discussions of design of details for marine engines and numerous examples drawn from actual practice. The distinctive feature of the book under review is really found in its exclusive devotion to details of design, whereas preceding text-books have been more comprehensive in scope, and as a consequence are larger and more expensive.

Prof. Bragg confines attention to marine engines of reciprocating types; he leaves untouched problems connected with steam turbines as applied to shippropulsion, although these are undoubtedly of primary importance in present circumstances. Rules and specimen calculations are given for determining the dimensions of cylinders, the "cut-off" in the admission of steam, the length of stroke, and other particulars for engines of specified powers and given rates of revolution. Steam speeds, valve diagrams, valves, and valve-gear are treated at considerable length. Attention is directed also to methods for calculating the strengths of many of the principal fixed and moving parts of reciprocating marine engines; in some cases the rules for details of marine engines issued by the principal societies established for the survey and classification of merchant shipping are reproduced.

The last two sections of the book contain a full discussion of the details of design for auxiliary engines fitted for the purpose of turning the crankshafts over-when valves are being set or when the main engines are being overhauled-and of designs for small engines used for reversing the main engines in steamships. About one-sixth of the total space in the volume is devoted to these subjects, although they are unquestionably of less relative importance than must be assigned to other matters, to which less attention has been devoted by the author. His hope and expectation, as expressed in the preface, are that by keeping the book "free from the descriptive part of the subject it will fill a decided void" and be "much more readily available" than other text-books "for the particular use for which it is intended." The majority of students will, in all probability, favour the use of text-books which are less restricted in aim, and are richer in descriptions and illustrations of various types of engines. This opinion, however, in no sense reflects on the manner in which the author has done his work; and, of course, he is entitled to form and act upon his own estimate of the kind of text-book likely to prove of most service to students of marine engineering.

W. H. W.

\section{OUR BOOK SHELF.}

Puppets: a Work-a-Day Philosophy. By George Forbes, F.R.S. Pp. ix $+\mathrm{r}_{3}$. (London: Macmillan and Co., Ltd., I9II.) Price $3 s$. 6 d. net.

Ttus is a philosophical sketch, or skeleton, clothed upon with the human interest of a story. A party of happy and well-to-do people are staying at a Scottish castle, and James Gordon expounds to them his philosophical views. These are of the idealistic kind, which may roughly be called Berkeleyan, and Gordon develops them very ingeniously, making some use of the modern psychological doctrine of the subconscious. All our knowledge of the world is a mental knowledge; all "things" are thoughts or, at least, cannot 